Supporting information for

\title{
Nanotemplate Fabrication of PEG-Containing Thermosetting Polyurethane Nanoarray toward Enhanced Thermal Storage
}

Shaoming Fang $t^{*}$, Cong Lit, Lijun Gaot, Quanwen Sunt, Jing Cuit, Liming Zhou ${ }^{*}$,

Caixia Yangt, and Haifeng Yu‡*

† Key Laboratory of Surface \& Interface Science of Henan Province, School of

Material and Chemical Engineering, Zhengzhou University of Light Industry,

Zhengzhou, Henan 450002, China.

*E-mail: mingfang@zzuli.edu.cn (S. Fang), zlming1212@126.com (L. Zhou)

$\ddagger$ Department of Material Science and Engineering, College of Engineering and Key

Laboratory of Polymer Chemistry and Physics of Ministry of Education, Peking

University, Beijing 100871, China.

*E-mail: yuhaifeng@pku.edu.cn (H. Yu) 


\section{Experimental section}

S1. Materials. Isophorone diisocyanate (IPDI) was purchased from Shanghai NCM Hersbit Chemical Enterprise. 2-Hydroxyethyl methacrylate (HEMA) was provided by Tianjin Chemical Reagent Research Institute. Polyethylene glycol (PEG) with molecular weights $\left(\mathrm{M}_{\mathrm{w}}\right)$ of 400, 600, and $1000 \mathrm{~g} \mathrm{~mol}^{-1}$ were purchased from Tianjin Fengchuan Chemical Reagent Technologies Co. Ltd, China. AAO templates with pore diameter range of 80-300 nm were purchased from $\mathrm{Pu}$-Yuan Nanotechnology Limited Company, China. Before used, the AAO templates were ultrasonically vibrated in ethanol and water for $15 \mathrm{~min}$, respectively, dried in the oven $\left(50{ }^{\circ} \mathrm{C}, 3\right.$ hours $)$. Dibutyltin dilaurate (DBTL) was obtained from Shanghai Yuming Industrial Co., Ltd. Yuanhang Reagent Factory, China. 2,2'-azobis (2-methylpropionitrile) (AIBN), obtained from Tianjin Fuchen Chemical Reagants Factory, China, was purified by recrystallization from ethanol before used. Other materials like ethanol, DMSO are standard laboratory reagents and used as received.

S2. Synthesis of macromonomer PUA (MPUA). In a typical example, the PEG400 was first reacted with IPDI (molar ratio 1:2), following the terminated reaction with HEMA (a molar ratio of 1:2) at room temperature. Take macromonomer PUA1 as an example, 13.34 g IPDI together with 12 g PEG400 (molar ratio of IPDI to PEG 2:1) were mixed in a 100-mL three-necked flask and kept stirring under dry nitrogen atmosphere for $10 \mathrm{~min}$. Then $0.016 \mathrm{mmol}$ DBTL was added as a catalyst, and the system was kept stirring at $25^{\circ} \mathrm{C}$ for $30 \mathrm{~min}$. After that, mixture of $0.12 \mathrm{~g}$ AIBN and 7.81 g HEMA was added to the system (molar ratio of HEMA to IPDI 1:1), and 
continue stirring below $20{ }^{\circ} \mathrm{C}$ for $30 \mathrm{~min}$. The obtained macromonomer was labeled as MPUA1, with both ends capped by allyl groups. The same procedure for PUA2 and PUA3.

The products of macromonomer PUA were labeled as PUA1, PUA2 and PUA3, respectively, according the PEG molecular weight (400, 600 and $1000 \mathrm{~g} / \mathrm{mol})$. Details information of the PUA are listed in Table S1.

S3. Synthesis of TPU bulk and nanoarrays. For detail, a certain amount of the macromonomer PUA was introduced into the AAO nanocavities and kept at a certain temperature for $30 \mathrm{~min}$ in order to seep into the nanocavities. During this process, the polymerization was thermally initiated and the in-situ polymerization occurred in the AAO nanocavities. Further curing reaction of MPUA took place under different temperature, 40,50 , and $60{ }^{\circ} \mathrm{C}$ for $1 \mathrm{~h}$, respectively, and $70{ }^{\circ} \mathrm{C}$ for $24 \mathrm{~h}$, and the TPU nanoarrays were obtained. Other TPU nanoarrays were prepared following the same procedure. The obtained TPU nanoarrays were labeled as TPU\#@N\#, according to their macromonomers and AAO nanotemplate diameters. For example, TPU1@N80 represents the macromonomer PUA1 (PEG molecular weight of 400) polymerized in AAO nanotemplate with a pore diameter of $80 \mathrm{~nm}$. Before measurement, the TPU were previously removed from the AAO nanocavities by dipping in $\mathrm{NaOH}$ solution (3 mol $\mathrm{L}^{-1}$ ) several times, then washed with deionized water. The chemical composites and details of TPU bulk and nanoarrays are listed in Table S1. Furthermore, the TPU nanoarrays for characterization were prepared by transfer the dilute dispersions of 
nanoarrays from ethanol onto silicon (Harbin Terbo Technologies Co., Ltd) or copper grid (Zhengzhou Yasheng Electronic Technology Co., Ltd), according to the characterization methods.

Table S1. The chemical composition for preparing MPUA

\begin{tabular}{cccccc}
\hline Sample & $\begin{array}{c}\text { IPDI } \\
(\mathrm{mol})\end{array}$ & $\begin{array}{c}\text { PEG } \\
\left(\mathrm{M}_{w}, \mathrm{~mol}\right)\end{array}$ & $\begin{array}{c}\text { HEMA } \\
(\mathrm{mol})\end{array}$ & Morphology & $\begin{array}{c}\text { Diameter } \\
(\mathrm{nm})\end{array}$ \\
\hline PUA1 & 0.04 & $400,0.02$ & 0.04 & Macromonomer & - \\
PUA2 & 0.04 & $600,0.02$ & 0.04 & Macromonomer & - \\
PUA3 & 0.04 & $1000,0.02$ & 0.04 & Macromonomer & - \\
TPU1@B & 0.04 & $400,0.02$ & 0.04 & TPU Bulk & - \\
TPU1@N80 & 0.04 & $400,0.02$ & 0.04 & TPU nanoarrays & 80 \\
TPU2@N100 & 0.04 & $600,0.02$ & 0.04 & TPU nanoarrays & 100 \\
TPU3@N100 & 0.04 & $1000,0.02$ & 0.04 & TPU nanoarrays & 100 \\
TPU3@N160 & 0.04 & $1000,0.02$ & 0.04 & TPU nanoarrays & 160 \\
\hline
\end{tabular}
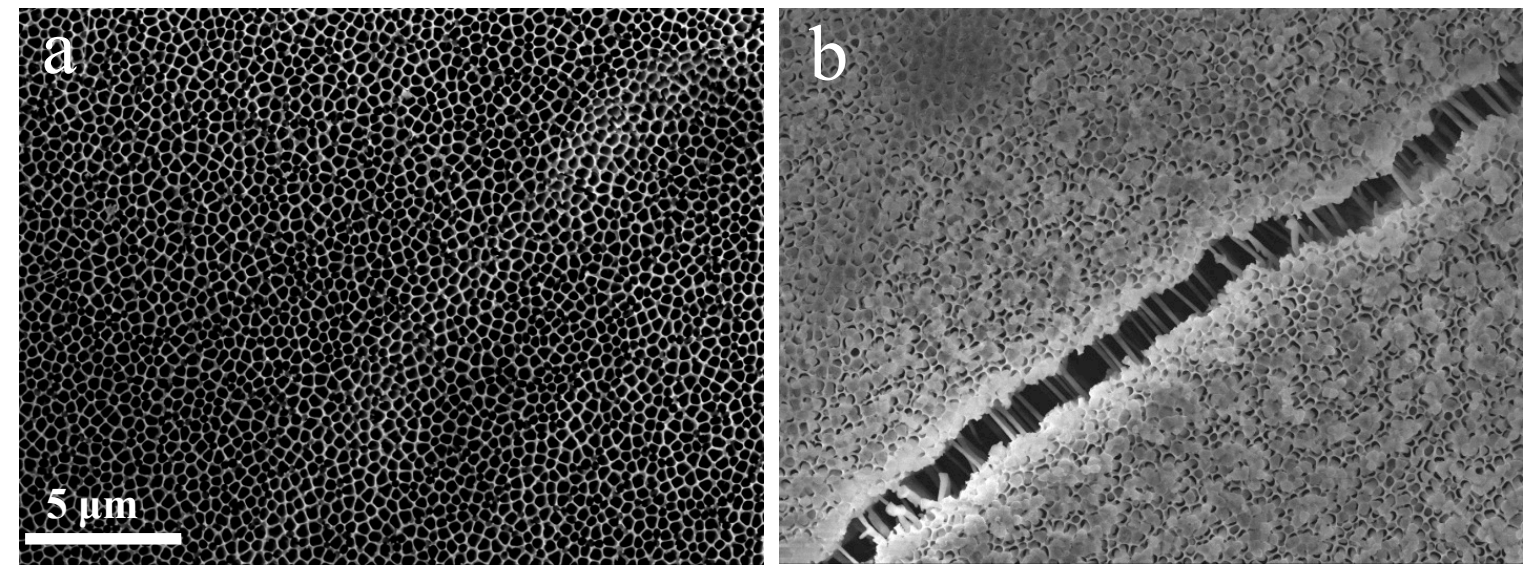

Figure S1. FESEM micrographs of AAO nanotemplates with nanopores of 160-200 $\mathrm{nm}$ : (a) top surface of the received nanotemplates and (b) bottom surface and the split nanotemplate after polymerization of the infiltrated macromonomer PUA1. 

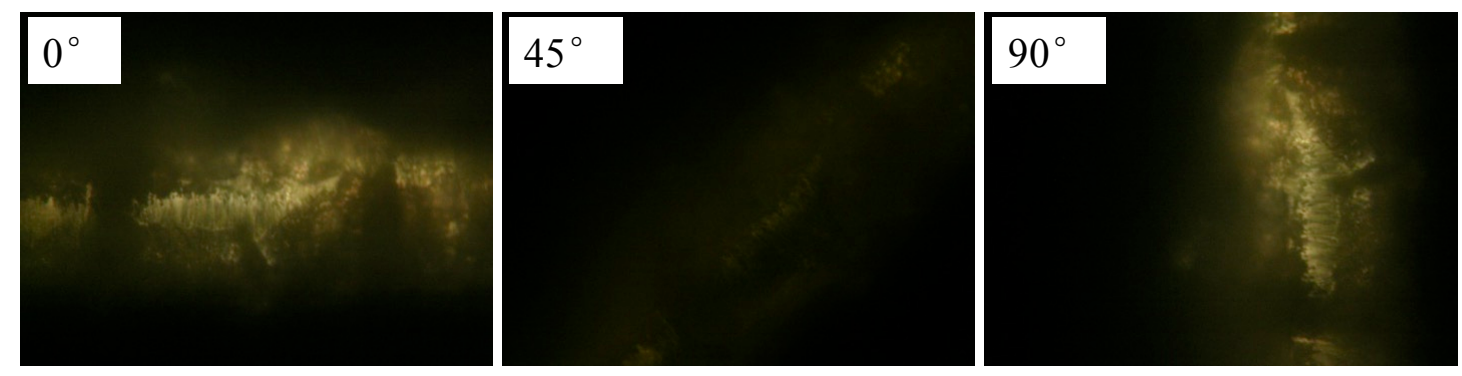

Figure S2. Photographs of TPU3@N200, as observed under a polarized optical microscope

Table S2. Comparison of the therma storage ability with other works.

\begin{tabular}{|c|c|c|c|c|}
\hline $\begin{array}{c}\text { Phase } \\
\text { Transition }\end{array}$ & Materials & $\begin{array}{c}\text { M }_{\mathrm{n}} \text { PEG } \\
(\mathrm{g} / \mathrm{mol})\end{array}$ & $\begin{array}{c}\text { Enthalpy } \\
\text { change }(\mathbf{J} / \mathbf{g})\end{array}$ & Reference \\
\hline Solid-Solid & PEG/PU & 1000 & 85.18 & 1 \\
\hline Solid-Liquid & PU & $\begin{array}{l}\text { PEG1000 } \\
\text {-TDI }\end{array}$ & 69.5 & 2 \\
\hline Solid-Solid & PU foams & $\begin{array}{l}\text { n-hexadecane } \\
\text { /PEG600 }\end{array}$ & 34.5 & 3 \\
\hline Solid-Solid & $\begin{array}{c}\text { TPU } \\
\text { nanoarrays }\end{array}$ & 1000 & 40.23 & This work \\
\hline
\end{tabular}

\section{References}

(1) Ke, G.-Z.; Xie, H.-F.; Ruan, R.-P.; Yu, W.-D. Preparation and Performance of Porous Phase Change Polyethylene Glycol/Polyurethane Membrane. Energy Convers. Manage. 2010, 51, 2294-2298.

(2) Alkan, C.; Günther, E.; Hiebler, S.; Ensari, Ö. F.; Kahraman, D. Polyurethanes as Solid-Solid Phase Change Materials for Thermal Energy Storage. Solar Energy 2012, $86,1761-1769$.

(3) Sarier, N.; Onder, E. Thermal Characteristics of Polyurethane Foams Incorporated with Phase Change Materials. Thermochimica Acta 2007, 454, 90-98. 